\title{
La construcción discursiva de la República de Chile
}

\author{
Miguel Metzeltin* \\ Universidad de Viena, Austria \\ Academia Chilena de la Lengua, Chile
}

\section{EL NACIMIENTO DE LOS ESTADOS NACIONALES}

Un Estado se puede concebir como una unión de un número considerable de personas que se juntan cooperando para garantizar el bienestar de una comunidad. Esta idea aparece por primera vez en la Política de Aristóteles y se transmite más o menos variada a lo largo de toda la tradición política occidental (Alfonso X el Sabio, Dante, Rodrigo Sánchez de Arévalo, etc.). Un Estado puede formarse y crecer a través de lentos procesos pluriseculares, como Suiza, o ser el resultado de una empresa consciente que lleva a una estructuración y jerarquización relativamente rápidas de la comunidad estatal. Estas estructuraciones y jerarquizaciones con sus tendencias homogeneizantes constituyen la base de los Estados nacionales modernos. En la época del absolutismo, se pueden observar en algunos Estados europeos como Francia claros esfuerzos por conseguir una homogeneización administrativa y religiosa. Pero todavía no se plantea claramente el problema de quién representa al Estado. Los reyes y los príncipes son en principio señores o dueños gobernantes de comunidades territoriales. El poder y

\footnotetext{
* Para correspondencia, dirigirse a: Miguel Metzeltin (michael.metzeltin@univie.ac.at), Universidad de Viena, Institut für Romanistik, Garnisongasse 13, A-1090 Wien, Austria.
} 
la administración del Estado se reparten entre la Corona y los Estados, estamentos o brazos que, reunidos como Cortes o Etats généraux, tienen cierta función de representación estatal, pero no designan al gobierno. Las Cortes tienen carácter más bien consultivo.

En Inglaterra, los vasallos del rey Juan sin Tierra, descontentos por las pesadas cargas que el rey les exigía, lo obligaron en 1215 a conceder la llamada Magna Charta (su título exacto es Concordia inter Regem Johannem et Barones pro concessione libertatum ecclesie et regni Anglie), una especie de contrato para regular las relaciones entre el rey y los vasallos. Esta carta constituye la base del desarrollo parlamentario y constitucional inglés. Bajo su sucesor, Enrique III (1216-1272), el Parlamento consiguió imponer cierta dominancia como instancia de consulta y de decisión. Fundamentalmente, se trataba de obligar al rey a no aumentar los impuestos sin previo acuerdo del Parlamento. Estos aumentos se debían a las necesidades financieras provocadas por las frecuentes guerras. Carlos I disolvió el Parlamento en 1629 , porque éste se resistía a ampliarle los derechos prerrogativos y porque no estaba de acuerdo con la reconciliación del rey con Roma. Pero en 1640, el rey fue obligado a reconvocar el Parlamento (Parlamento largo, 16401660), que ahora solo podía ser disuelto por acuerdo de sus miembros, y se aseguró el control de la legislación sobre los impuestos. A partir de entonces, rey y Parlamento solo podían gobernar juntos. Pero, en realidad, todavía no se puede hablar de un Parlamento verdaderamente representativo, porque éste está exclusivamente en las manos de la alta nobleza y de la Gentry (baja nobleza).

En Francia, los Estados Generales eran asambleas más bien excepcionales convocadas por el rey con motivo de crisis políticas o financieras. Estaban formadas por representantes del clero, de la nobleza y de las ciudades (Tercer Estado), se reunían por estamentos y el voto era estamental, con la consecuencia de que el Tercer Estado siempre quedaba en situación inferior ante la nobleza y el clero. Los Etats généraux fueron convocados por primera vez en 1302 por Philippe le Bel para legitimizar su poder frente a las pretensiones teocráticas del papa Bonifacio VIII. Repetidas veces intentaron controlar las decisiones del rey, como en 1357, cuando fueron convocados por el delfín Charles durante la prisión de su padre Jean le Bon. Pero, fundamentalmente, era asambleas consultivas y no legislativas. Los diputados representaban a los estamentos, no al pueblo en general. El paso hacia un verdadero parlamento representativo solo se dio debido a las grandes transformaciones sociales en Francia y, más concretamente, a la conquista del poder en 1789 por el Tiers Etat, tan claramente descrito por Emmanuel Sieyès en su Qu'est-ce que le Tiers état? A través de su autopresentación como nación y a través de la abolición de los estamentos empezó el desarrollo 
propiamente dicho de los Estados nacionales modernos, en que el pueblo se transformó en nación y los súbditos en ciudadanos. Gracias, sobre todo, a las ideas del Contrat social (1762), de Jean Jacques Rousseau, con la fusión de las voluntades individual y general, el Estado administrativo absolutista se transformó en Estado constitucional.

\section{LA INVENCIÓN METADISCURSIVA DE LOS ESTADOS NACIONALES}

Los Estados europeos actuales, como también muchos otros Estados nacionales y, más en general, las grandes comunidades "regionales" son invenciones de la mentalidad liberal y democrática de los siglos XIX y XX que tienen sus raíces en la Revolución Francesa. Han sido concebidos de manera más o menos consciente para gobernar determinados territorios de manera centralista y racional, pero al mismo tiempo democrática. Eric Hobsbawm y Terence Ranger hablan en este contexto de The Invention of Tradition (1983), Benedict Anderson de Imagined Communities (1983), Richard White de Inventing Australia (1981), Imman Fox de la Invención de España (1997) y la búlgara Maria Todorova analiza la imagen europea de los Balcanes en un libro titulado Die Erfindung des Balkan (1999). Ya en 1931, el historiador americano T. W. Riker había hablado de The Making of Roumania. Como nos enseña la historia, los grupos que forman la base del nuevo Estado nacional tienen que ser preparados para comprender mental y semióticamente estas invenciones, de manera que éstas puedan adquirir cierta unidad, sin la cual no podrían funcionar. Pues, como observa el historiador italiano Ernesto Galli della Loggia (1998: 157-158), una identidad nacional y su sentimiento no son fenómenos naturales:

[...] come tali l'identità nazionale e il suo sentimento non esistono in natura. L'una e l'altro sono il prodotto di élite ideologico-culturali, in genere inserite nelle istituzioni dello Stato [...]. È la doppia azione combinata di élite ideologico-culturali del genere e delle istituzioni statali - soprattutto di quelle preposte all'amministrazione concreta dell'interesse generale (per esempio il fisco o la giustizia), ovvero, specificamente, alla formazione di una cultura dell'appartenenza collettiva (como sono la scuola e l'esercito) - è siffatta azione combinata che è all'origine dell'identità nazionale e del relativo sentimento. 
Si observamos la formación de los Estados europeos y americanos a partir del siglo XIX, podemos proponer como hipótesis que la invención de los modernos Estados nacionales se constituye más o menos cronológicamente a través de varios procesos semióticos y mitificadores. Estos procesos constituyen el metadiscurso del Estado nacional o Estado-nación (el binomio sugiere una estrecha relación entre política e identidad poblacional) y se pueden ordenar según la siguiente "trama" narrativa:

- $\quad$ Toma de conciencia

- Territorialización

- Historización

- Estandarización e historización de una lengua nacional

- Creación de una literatura nacional

- Institucionalización

- Medialización

- Globalización

Estos procesos se desarrollan de manera fuertemente discursiva a través de diferentes géneros textuales y otros productos semióticos. La repetida experiencia común de estos procesos crea una identidad nacional estatal que lleva a un sentimiento de protección y seguridad colectiva. Consecuencia natural de la identidad nacional es el establecimiento de la ciudadanía, que da acceso a los recursos estatales (nacionales). Por eso se puede también decir que la creación de Estados nacionales se basa en la concepción de cómo se pueden distribuir los recursos entre grandes poblaciones. El Estado nacional con su organización tiene su máxima expresión semiótica en su constitución. A continuación, reconstruiremos el "metadiscurso" de la formación nacional de Chile.

\section{TOMA DE CONCIENCIA}

Un grupo, gracias a su elite, toma conciencia de sí mismo categorizándose a sí mismo como pueblo o nación y dándose un nombre, caracterizando al mismo tiempo a otros grupos como pueblos o naciones (autopercepción, heteropercepción, estereotipación), y se afirma en el interior y en el exterior para la realización de sus propios intereses. De esta manera, se crea una voluntad política común y una conciencia nacional. El desarrollo de la 
percepción identificadora se produce a menudo a través de guerras (los franceses contra los ingleses, los castellanos contra los moros, los italianos contra los austríacos, etc.) y, semióticamente, a través de la recitación y transmisión de cantares de gesta.

La toma de conciencia de una identidad propia se desarrolló probablemente entre la elite de Santiago, en el siglo XVII, en oposición a Lima y por la posición geográfica periférica de la Capitanía General de Chile. En el siglo XVII, tenemos el testimonio del jesuita Manuel Lacunza, exiliado a Italia, quien expresa su chilenidad en una carta dirigida a su abuela: "Todos los chilenos, y yo el primero, pedimos este bien para nuestra patria" (Carey, Irarrázaval y Piñera 1999: 18). La autopercepción y la heteropercepción aparecen evidentes en el discurso que Bernardo O'Higgins dirige a sus tropas en el camino a Chacabuco: "Compatriotas y amigos: el numen de la libertad me restituye por fin al suelo patrio. (...) La dulce patria, el hermoso Chile, vuelve a ocupar el rango de nación. (...) Arrojemos al grupo miserable de españoles advenedizos, que dos años ha vulneran nuestro honor, detentan nuestros bienes e insultan con cruel impavidez a todo americano. (...) Chilenos: yo os juro morir o libertaros" (Irarrázaval y Piñera 1999: 13). En la tira cómica de Von Pilsener, historieta publicada en el semanario Zig-Zag en 1906 y 1907, Pedro Subercaseaux (con el seudónimo Lustig) contrasta las imágenes estereotipadas del alemán y del chileno.

\section{TERRITORIALIZACIÓN}

La comunidad que ha tomado conciencia de sí misma inventa y define un territorio, buscando un nombre por todos reconocido, estableciendo supuestas fronteras naturales o históricas y alabando la fertilidad y la belleza del país. Famoso es el Loor de España que ya en el siglo XIII Alfonso el Sabio introdujo en su Coronica de Espanna (cap. 311). Función laudatoria tienen hoy los libros de ilustraciones que, de manera panorámica, presentan los logros, los adelantos y las regiones de un Estado. A la invención del territorio pertenecen también sus representaciones cartográficas, el establecimiento de lugares emblemáticos como el Santuario de Covadonga para los españoles o el Mosteiro da Batalha para los portugueses, y la declaración constitucional de su indivisibilidad.

La conciencia que une una territorialidad chilena aparece ya bajo el primer gobernador, Pedro de Valdivia, quien, dirigiéndose en 1545 al emperador 
Carlos V respecto a "estas (sc. provincias) de la Nueva Extremadura, llamadas primero Chili" le informa que "esta tierra es tal, que para poder vivir en ella y perpetuarse no la hay mejor en el mundo; dígolo porque es muy llana, sanísima, de mucho contento". También el poeta y soldado español Alonso de Ercilla y Zúñiga, que estuvo en Chile entre 1557-1559, alaba el país en su poema épico La Araucana (1569-1589): "Chile, fértil provincia y señalada, / en la región antárctica y famosa" (Parte I, Canto I). En 1646, el jesuita Alonso de Ovalle, que había nacido en Chile, publica en Roma una Historica relacion del Reyno de Chile, en que se encuentra también una Tabula geographica Regni Chile.

Ya en el siglo XIX, el naturalista francés Claudio Gay, por encargo del ministro Diego Portales, redacta una monumental Historia física y política de Chile (28 volúmenes y 2 atlas, 1844-1871); Amadeo Pissis publica después de una preparación de veinte años una Geografía Física de Chile (1876) y Francisco Solano Asta-Buruaga y Cienfuegos su Diccionario geográfico de la República de Chile (18992). En 1903, el editor Carlos Tornero en Santiago presenta en gran formato un espléndido volumen panorámico titulado Chile. Descripción física, política, social, industrial y comercial de la República de Chile ilustrada con numerosos grabados y mapas. La especial geografía de Chile es realzada en el popular libro Chile o una loca geografia (1940), de Benjamín Subercaseaux, y cantada en varios textos del poemario Poema de Chile (1967, póstumo), de Gabriela Mistral. El territorio es tematizado también en pintura, como Frutos del País, de Bruna Truffa (1963-), y en discursos políticos, como en el conocido Discurso de la Patria Joven, de Eduardo Frei Montalva, pronunciado el 21 de junio de1964:

Ustedes, muchachos del norte traen la lección del heroísmo. En sus pies hay sal de la pampa y polvo del desierto y en vuestra piel, impregnados, el cobre y el hierro, el salitre y la plata. Es el norte que llega. Ustedes, muchachos del sur, con sus canciones, han conmovido a las viejas araucarias y a los milenarios alerces, cuyos troncos calcinados parecen al viajero cementerios de héroes antiguos. Traen ustedes en su mirada los lagos, los ríos y los bosques, y en sus manos, los frutos de nuestra tierra. Ustedes han venido flanqueados por dos compañeros: la cordillera y el mar, que nunca abandonan al chileno. Y ustedes nos traen una lección. La lección de esta tierra, de este territorio chileno que nos ama, que busca y espera nuestro amor como un gran amor, como un gran amigo.

Desde la constitución del nuevo Estado de Chile, los políticos cuidan la integridad territorial, anclándola en las constituciones: 
“Artículo 3. El territorio de Chile conoce por límites: al Sur, el Cabo de Hornos; al Norte, el despoblado de Atacama; al Oriente, los Andes; al Occidente, el mar Pacífico. Le pertenecen las islas del archipiélago de Chiloé, las de la Mocha, las de Juan Fernández, la de Santa María y demás adyacentes" (Constitución política del Estado de Chile, 1822).

"Art. 1. El Estado de Chile es uno, e indivisible" (Constitución política de Chile, promulgada el 29 de diciembre de 1823).

\section{HISTORIZACIÓN}

A través de una selección y configuración conscientes de hechos históricos, los historiadores, los pintores y los etnólogos del grupo inventan y construyen una historia "nacional", porque se considera que un pueblo sin historia no puede constituir una nación. Con ello se insiste en que el grupo constituye una nación autónoma o independiente, unitaria y organizada, que luchó por su autonomía o su libertad, que ha renacido -piénsese en el Risorgimento italiano-y que tiene una historia antigua. Consecuentemente, se escriben y componen obras historiográficas, óperas y novelas, y se pintan cuadros que tematizan la continuidad de la nación y ponen en escena momentos considerados relevantes para su formación. A menudo se hace empezar la historia nacional de manera anacronística mucho antes de la fundación del Estado nacional. Así se crea y propaga una historia "mítica" de la nación. Una forma particular de historización es la recolección y recuperación de tradiciones populares.

La independencia es uno de los temas repetidos en los escritos políticos del nuevo Estado chileno. Así se puede leer en El catecismo de los patriotas, que apareció en el periódico El Monitor Araucano (núm. 99), del 27 de noviembre de 1813: "La libertad nacional es la independencia; esto es, que la Patria no dependa de la España, de la Francia, de Inglaterra, de Turquía etc., sino que se gobierne por sí misma". El 12 de febrero de 1818, O'Higgins proclama en Talca la independencia nacional: "Juro a Dios y prometo a la patria bajo la garantía de mi honor, vida y fortuna, sostener la presente declaración de independencia absoluta del Estado chileno, de Fernando VII, de sus sucesores y de cualquier otra nación extraña", "Hemos tenido a bien (...) declarar (...) a la gran confederación del género humano que el territorio continental de Chile y sus islas adyacentes forman de hecho y por derecho un Estado libre, independiente y soberano, y quedan por siempre 
separados de la monarquía de España" (Acta de Independencia de Chile). En el mismo año aparece en Santiago el Manifiesto que hace a las naciones el Director Supremo de Chile de los motivos que justifican su revolución y la declaracion de su independencia. El tema de la independencia preocupa a los chilenos hasta nuestros días. El 4 de setiembre de 1970, el nuevo presidente Salvador Allende pronunció un discurso en que anunciaba: "somos los herederos legítimos de los Padres de la Patria, y juntos haremos la segunda independencia: la independencia económica de Chile" (Irarrázaval y Piñera 1999: 139).

Las primeras obras historiográficas se deben a la pluma de José Victorino Lastarria (Historia constitucional de medio siglo, 1853), Miguel Luis Amunátegui (La dictadura de O'Higgins, 1853), Diego Barros Arana (Historia jeneral de la independencia de Chile, 1854-1858) y Benjamín Vicuña Mackenna (Historia de los diez años de la administración de don Manuel Montt, 1862). Narciso Desmadril edita, en 1854, en Santiago de Chile, el primer volumen de una Galeria nacional o Coleccion de biografias i retratos de hombres celebres de Chile. Escrita por los principales literatos del pais. Diego Barros Arana publica una monumental Historia jeneral de Chile en 16 tomos (1884-1902) que va a ser el modelo de la historiografía chilena, en la opinión del historiador Francisco Antonio Encina: "uno de los mayores esfuerzos de investigación que se haya realizado en América". Entre los historiadores chilenos existe también cierta tendencia a iniciar la historia del país en la prehistoria o muchos antes de la creación del Estado nacional. Francisco Antonio Encina escribe una Historia de Chile desde la prehistoria hasta 1891 (20 tomos, 1942-1952). José Toribio Medina edita, entre 1888 y 1902, los 30 tomos de la Colección de documentos inéditos para la historia de Chile (relativos al siglo XVI). Pedro Subercaseaux (18801956) ilustró varios momentos y personajes de la historia de Chile en sus pinturas (Descubrimiento de Chile por Almagro, 1918; Batalla de Maipú, 1904; Jura de la Independencia, 1945; Bernardo O'Higgins Riquelme; Manuel Baquedano, etc.).

En 1909, se funda la Sociedad de Folklore Chileno, que edita la Revista de Folklore Chileno. Ya en su juventud, el etnólogo Ramón A. Laval (1862-1929) había empezado a recoger cuentos populares; en 1910, publica los Cuentos Chilenos de nunca acabar y, en 1923, los Cuentos Populares de Chile. Julio Vicuña Cifuentes (1865-1936) publica, en 1912, los Romances populares y vulgares recogidos de la tradición oral chilena. 


\section{ESTANDARIZACIÓN E HISTORIZACIÓN DE UNA LENGUA NACIONAL}

Escogiendo una variedad lingüística hablada en el ámbito nacional, dándole reglas explícitas y homogeneizándola (mediante ortografías, gramáticas, diccionarios, manuales de poética y retórica), los gramáticos del grupo crean una lengua nacional con su propia historia. Normalmente, se resalta la unidad de esta lengua en detrimento de las demás variedades y se oficializa. En el caso de lenguas policéntricas como el español, se pueden realzar las peculiaridades del país respectivo. Si ya existe una lengua estandarizada, se subrayan su antigüedad y su pujanza, y se fomenta su cultivo a través de instituciones especiales.

Cuando Chile se independizó, el castellano ya había sido estandarizado desde hace tiempo, en particular, por la Real Academia Española (RAE). En un país tan lejano de España como Chile era por eso importante insistir en el cultivo de la lengua considerada propia. En su discurso de inauguración de la Universidad de Chile (1843), su primer rector, Don Andrés Bello, propone, entre otras cosas:

[...] el estudio de nuestra lengua me parece de una alta importancia. Yo no abogaré jamás por el purismo exajerado que condena todo lo nuevo en materia de idioma; creo por el contrario, que la multitud de ideas nuevas que pasan diariamente del comercio literario a la circulación general, exige voces nuevas que las representen (Irarrázaval y Piñera 1999: 26).

El mismo Bello publica en 1847, en Santiago, una Gramática de la lengua castellana destinada al uso de los americanos, hasta el día de hoy una de las mejores gramáticas de la lengua española, en cuyo prólogo defiende el uso de las variantes americanas:

No se crea que recomendando la conservación del castellano sea mi ánimo tachar de vicioso y espurio todo lo que es peculiar de los americanos. Hay locuciones castizas que en la Península pasan hoy por anticuadas y que subsisten tradicionalmente en Hispano-América ¿por qué proscribirlas? Si según la práctica general de los americanos es más analógica la conjugación de algún verbo, ¿por qué razón hemos de preferir la que caprichosamente haya prevalecido en Castilla? Si de raíces castellanas hemos formado vocablos nuevos, según los procederes ordinarios de derivación que el castellano reconoce, y de que se ha servido y se sirve continuamente para aumentar su caudal, ¿qué motivo hay para que nos 
avergoncemos de usarlos? Chile y Venezuela tienen tanto derecho como Aragón y Andalucía para que se toleren sus accidentales divergencias, cuando las patrocina la costumbre uniforme y auténtica de la gente educada.

En 1885, se fundó la Academia Chilena de la Lengua, hoy integrada en la Asociación de Academias de la Lengua Española; en sus publicaciones, como el Boletín de la Academia de la Lengua (desde 1915) se ocupa del uso y de la evolución del español en Chile. En 1978, la Academia publicó un Diccionario del habla chilena, pronto agotado. En 2010, se acaba de publicar, bajo la dirección del académico Alfredo Matus Olivier, el Diccionario de uso del español de Chile (DUECH).

\section{CREACIÓN DE UNA LITERATURA NACIONAL}

Creando continuamente nuevos textos, originales o traducidos, valorizándolos y seleccionándolos, los literatos del grupo inventan una literatura nacional con autores y textos considerados relevantes para la imagen de la nación y, por lo tanto, canónicos. Surgen así manuales de historia de la literatura y antologías de textos.

El nuevo Estado chileno tiene que inventar una literatura propia de raíz. La primera actividad "literaria" es un vivo periodismo. En los primeros años de la república aparecen entre otros, La Aurora de Chile (1812, redactor Camilo Henríquez), El Monitor Araucano (No. 1, martes 6 de abril de 1813), la Gazeta Ministerial del Gobierno de Chile (noviembre de 1815, redactor: Fr. José María de la Torre), la Gaceta del Supremo Gobierno de Chile (No. 1, 26 de febrero de 1817, Santiago). El Mercurio chileno de 1828-1829 publica numerosos artículos literarios. Bajo el influjo del español José Joaquín de Mora y del venezolano Andrés Bello se forma hacia 1840 una primera generación de literatos con carácter chileno: José Victorino Lastarria, Francisco Bilbao, Juan Bello, Santiago Lindsay, Jacinto Chacón, entre otros. Éstos fundan en 1842 la Sociedad Literaria, en la que Lastarria presenta un programa para la creación de una literatura nacional. Santiago Lindsay escribe poesías patrióticas, Jacinto Chacón, el poema "progresista" Historia Moderna, Lastarria, el cuento El mendigo (1843), que designa como ensayo de novela histórica. La historia de la literatura empieza con las Nociones de historia literaria universal, de Diego Barros Arana (1869), la Historia de la literatura colonial de Chile, de José Toribio 
Medina (1878), los Recuerdos literarios. Datos para la historia literaria de la América española $i$ del progreso intelectual en Chile (1878/1885), de José Victorino Lastarria y el Bosquejo histórico de la literatura chilena, de Domingo Amunátegui Solar (1915).

El gran nivel literario alcanzado por las letras chilenas ha sido reconocido internacionalmente por la atribución del Premio Nobel de Literatura a Gabriela Mistral (1945) y a Pablo Neruda (1971). En las palabras de despedida pronunciadas por Radomiro Tomic con motivo de la muerte de Gabriela Mistral, el político chileno subrayaba "la asombrosa identificación del pueblo chileno con esta mujer triste y solitaria" y el simbolismo nacional de la literatura: "Ella es ahora (...) símbolo de esta comunidad de origen y destino de nuestra patria y preciosa salvaguardia de la identidad esencial de todos nosostros, en el gran regazo unificador de la nación", "un símbolo de Chile, una forma transfigurada de su pueblo", "el rostro multitudinario y el alma perdurable de su nación" (Irarrázaval y Piñera 1999: 111-116).

\section{INSTITUCIONALIZACIÓN}

Después de haber inventado una autoconciencia, un territorio, una historia, una lengua y una literatura, el grupo puede institucionalizarse de manera más explícita como Estado nacional:

- creando sus instituciones fundamentales (Parlamento y jefatura del Estado);

- $\quad$ proclamando su independencia absoluta;

- dándose una constitución;

- estableciendo una capitalidad;

- creando sus insignias o iconos emblemáticos (nombre oficial, bandera, escudo, himno, moneda);

- nacionalizando varias instituciones de interés público (enseñanza, comunicaciones, seguridad).

En los discursos se resalta que se tiene una patria común con la cual se es leal y que hay que defender. Surgen así odas a la patria y poesías que cantan el valor de los propios soldados.

En Chile, el primer Congreso Nacional fue inaugurado ya el 4 de julio de 1811, el cargo de jefe de Estado, con el título de Director Supremo, fue 
establecido por primera vez en 1814. La guerra contra los realistas por la independencia de Chile duró desde 1813 hasta 1826, pero ya el 12 de febrero de 1818 pudo tener lugar en Santiago la ceremonia solemne de proclamación y jura de la independencia. En esta ocasión fue izado el nuevo tricolor (Bandera Nacional: azul - blanco - rojo). Los primeros ensayos para la creación de un Escudo Nacional se remontan al año 1812, el escudo actual se establece en 1834 con dos animales emblemáticos para el país, el cóndor y el huemul. A petición del Director Supremo Bernardo O'Higgins, el argentino Bernardo de Vera y Pintado escribe en 1820 un himno nacional para el cual el chileno Manuel Robles compuso la música; ésta fue sustituida por una nueva del español Ramón Carnicer, ejecutada por primera vez en Santiago en 1828; en 1847, manteniendo la misma música, Eusebio Lillo Robles escribió el texto de la actual Canción Nacional. El nombre del país, (República de) Chile, gracias a su indiscutida tradición y pese a su no aclarada etimología, se mantuvo. En 1817, con la Patria Nueva, se reemplazó también el real colonial por el peso chileno. La capitalidad de Santiago también se mantuvo. Muy pronto, los nuevos gobernantes elaboran varios textos constitucionales (Reglamento Provisional de la Junta Gubernativa, 1810; Reglamento para el arreglo de la Autoridad Ejecutiva Provisoria de Chile, 1811; Reglamento Constitucional Provisorio, 1812; Reglamento para el Gobierno Provisorio, 1814; Constitución Provisoria para el Estado de Chile, 1818; Constitución política del Estado de Chile, 1822; Constitución politica y permanente del Estado de Chile, 1823; Leyes Federales, 1826; Constitución política de la república de Chile, 1828), hasta la Constitución de la República de Chile de 1833, la cual con su fuerte presidencialismo va a resultar adecuada para el país y solo será sustituida por la Constitución Política de 1925. Y ya en 1812 aparece en la Aurora de Chile del 4 de junio una primera Oda a la patria (de Camilo Henríquez).

\section{MEDIALIZACIÓN}

La nación-Estado concreta, difunde y reactualiza la común cultura nacional más o menos estereotipada a través de las escuelas públicas, los libros de texto, los medios de comunicación de masas, la organización de fiestas nacionales, la erección de monumentos conmemorativos y de edificios representativos y la formación de equipos nacionales. Así se crea y se establece un horizonte común de interpretación. 
Ya bajo el primer gobierno de Ramón Freire Serrano (1823-1826), se declaró Día Nacional no el día de la proclamación de la Independencia por Bernardo O'Higgins (12 de febrero de 1818), sino el día en que el Cabildo Abierto eligió la primera Junta de Gobierno en Santiago (18 de setiembre de 1810). El político Diego Portales (1793-1837) realzó la importancia de esta fiesta estableciendo que su organización es de la responsabilidad del Presidente de la República. Esta conmemoración se desarrolla como Fiestas Patrias con izamiento de la bandera, un solemne Tedeum, la parada militar, fuegos artificiales, luminarias, fondas y ramadas con bailes típicos (cuecas), bebidas y comidas tradicionales (chicha, mistela, vino; fierritos y empanadas), volantines, juegos tradicionales (destrezas campesinas, rodeos, carreras a la chilena). La Conferencia Episcopal instituyó en 1971 el Día de Oración por Chile (último domingo de setiembre); desde 1995 se celebra la Semana de la Chilenidad.

Ya en el año 1818 aparecía en el El Sol de Chile un largo artículo sobre la Instrucción nacional en que el autor anónimo afirmaba: "Nada interesa tanto a las naciones para conservar su libertad y defender sus derechos, como la instrucción de todos los ciudadanos (...). La educación es quien debe dar a las almas el carácter nacional”. En el año 1843, extinguida la Real Universidad de San Felipe, se inauguró la Universidad de Chile, la nueva guía de la educación superior del país. En 1849 abre sus puertas la Academia de Pintura en Santiago. Bajo los gobiernos de Manuel Bulnes (1841-1851) y de Manuel Montt (1851-1861) se fundan en ciudades y pueblos muchas escuelas primarias. El parlamentario Enrique Mac Iver sigue insistiendo en un discurso pronunciado el primer de agosto de 1900: "Pienso que no hay negocio público en Chile más trascendental que éste de la educación de las masas populares" (La crisis moral de la República).

A partir de la segunda mitad del siglo XIX, se erigen monumentos en recuerdo de importantes personalidades nacionales, como la estatua del héroe mapuche Caupolicán (por Nicanor Plaza, en el Cerro de Santa Lucía en Santiago, 1863) y la del libertador de Chile Bernardo O'Higgins (por Roberto Negri, en la Plaza de Armas de Chillán, 1919). Con ocasión de la celebración del primer Centenario de la República se construyeron la Biblioteca Nacional, la Estación Mapocho y el Museo Nacional de Bellas Artes (1910). 


\section{GLOBALIZACIÓN}

El Estado nacional se globaliza afirmándose e integrándose en la comunidad internacional, lo que consigue entre otras cosas:

- $\quad$ participando en tratados, conferencias y proyectos internacionales;

- fundando universidades y otras instituciones científicas;

- confeccionando grandes enciclopedias nacionales que recogen por un lado todo el saber sobre un país; por otro lado lo integran en el general saber humano;

- dando a conocer en el extranjero los grandes logros intelectuales y artísticos del país (a través de exposiciones, traducciones, etc.).

Los Estados Unidos deciden, en 1822, reconocer la independencia de todos los Estados que se habían separado de la monarquía española. España reconocerá a Chile como Estado libre independiente solo en 1844. Desde 1969, el Observatorio de La Silla colabora con la Organización Europea para la Investigación Científica en el Hemisferio Austral (ESO). Ya hemos visto que el gobierno instituye en 1843 la nueva Universidad de Chile. Contrariamente a Argentina y México, Chile no ha conseguido hasta ahora confeccionar una enciclopedia nacional; sin embargo, en 2001 la Editorial Ercilla de Santiago editó un Diccionario enciclopédico ilustrado en 12 fascículos. En el año 1964, se funda el Instituto de Chile con seis Academias (Lengua, Historia, Ciencias, Ciencias Sociales, Políticas y Morales, Medicina y Bellas Artes), dependiente del Ministerio de Educación Pública. La literatura chilena se ha hecho internacionalmente conocida gracias a los Premios Nobel concedidos a Gabriela Mistral (1945) y a Pablo Neruda (1971).

\section{LA CONCIENCIA NACIONAL}

Los Estados nacionales y las conciencias nacionales no son esencias, son constructos metadiscursivos realizados a través de procedimientos semióticos que dan a sus portadores un sentimiento de solidaridad y de seguridad y los hacen ciudadanos partícipes de las potencialidades de la nación. Para dar cierta estabilidad a estos constructos, hay que difundir y repetir continuamente los procesos semióticos que hemos indicado. A pesar de eso, toma de conciencia, territorialización, historización, estandarización 
e historización de una lengua nacional, creación de una literatura nacional, institucionalización, medialización, globalización son como tejidos cambiantes, que según el punto de vista y el momento histórico pueden tomar reflejos diferentes. Como observa justamente Jorge Larraín (2001: 274): "Es necesario partir de la base de que la identidad nacional no fue constituida de una vez para siempre en un pasado remoto, sino que se va construyendo en la historia con nuevos aportes".

Los posibles puntos de vista diferentes que se pueden adoptar respecto al metadiscurso de la formación de una nación quedan bien reflejados en la siguiente reflexión de Samuel Silva sobre el concepto de "patria" publicada en el suplemento El Sábado de El Mercurio, del 18 de setiembre de 1999:

$\mathrm{Ah}$, la patria. Es curioso tener una patria pequeña y provinciana cuando se vive en el corazón del imperio. Es curioso y estimulante, porque obliga a preguntarse qué significa realmente esa palabra tan grande. La patria es la bandera y el mapa, pero ¿qué más? ¿Qué estamos diciendo cuando decimos que somos chilenos? ¿Que somos una manera de hablar el castellano y el fantasma constante de los terremotos? ¿Que somos las machas a la parmesana y las discusiones políticas que terminan a gritos? Todo eso somos, claro, y más. Somos el legado de O’Higgins y Portales y, aunque nos duela, somos el legado de Allende y Pinochet. Somos cerros infranqueables, el global complementario, la obsesión por las apariencias, el mar helado y belicoso, el festival de Viña, el miedo al ridículo, las puestas de sol y la nulidad matrimonial. Somos todo eso. Y algunos creen -unos pocos- que somos también la copia feliz del edén y el asilo contra la opresión. Pero también somos esos símbolos abstractos, la bandera y el mapa.

\section{REFERENCIAS BIBLIOGRÁFICAS}

Carey, Alejandrina, Guadalupe Irrarázaval y Magdalena Piñera (comp.). 1999. Chile. Cartas con historia. Santiago de Chile: Los Andes.

Galli della Loggia, Ernesto. 1998. L'identità italiana. Bologna: il Mulino.

Irarrázaval, Guadalupe y Magdalena Piñera (comp.). 1999. Chile. Discursos con historia. Santiago de Chile: Los Andes.

LARRAín, Jorge. 2001. Identidad chilena. Santiago: LOM.

Metzeltin, Miguel, Thir, Margit (eds.), 2004. El poder. Análisis del discurso político español e hispanoamericano. Wien: Instituto Cervantes.

Metzeltin, Michael, Wallmann, Thomas. 2010. Wege zur europäischen Identität., Individuelle, Nationalstaatliche und supranationale Identitätskonstrukte. Berlin: Frank \& Timme.

Villalobos, Sergio. 1980-1999. Historia del pueblo chileno. Santiago de Chile: Zig-Zag/ Editorial Universitaria. 